\title{
Austin y Searle: la relación entre verbos y actos ilocucionarios
}

\author{
Marco Antonio Alarcón S*
}

\section{Resumen:}

El presente artículo intenta dar cuenta de la relación entre los actos de habla y la semántica a partir de los criterios utilizados por Austin y Searle en la elaboración de sus respectivas taxonomías de actos ilocucionarios. Para esto, se rastrean bibliográficamente algunos de los conceptos que utiliza Austin en sus conferencias de Harvard de 1955 (Austin, 1996) con la pretensión de intentar desambiguar en parte los criterios utilizados en la elaboración de sus categorías de verbos ilocucionarios. Luego, se revisan algunos conceptos de Searle y su taxonomía de actos ilocucionarios descritos en "Actos de Habla" (Searle, 1994); y desarrollados en "Una taxonomía de los actos ilocucionarios"(Searle, 1991b). Finalmente, a partir de la revisión de las clasificaciones y la discusión de la idea de significado que sustenta cada autor se obtiene que el significado de los verbos no depende solamente de su constitución léxica dada, sino que además se potencia en la interacción o uso de una expresión en una emisión real, en que se constituye como acto ilocucionario.

Palabras Clave: Acto de habla, Fuerza ilocucionaria, Verbos ilocucionarios, Significado

\section{Abstract:}

The present article reviews the criteria used by Austin and Searle on elaborating their respective taxonomies of illocutionary acts to account for the way in which speech acts and semantics relate both authors. In addition, some concepts of Searle and his taxonomy described in "Speech Acts" (1994) and developed in "A taxonomy of Illocutionary acts" (1991) are reviewed. Finally, the idea of meaning sustained by each author is discussed.

Key words: Speech Act - Illocutionary force - Illocutionary verbs - meaning

\footnotetext{
Profesor de Estado en Castellano, Magíster en Lingüística Universidad de Santiago de Chile, Profesor Adjunto Universidad Católica Silva Henríquez, Carrera de Pedagogía en Castellano, Carrera de Pedagogía en E. Básica. Profesor Adjunto Carrera de Fonoaudiología Universidad del Mar.
} 


\section{Introducción}

La problemática que identifica Austin (1996) -conciliar los actos de habla con la semántica-y que luego continúa Searle (1991a, 1991b, 1994) y otros filósofos del lenguaje es la base de una de las cuestiones claves dentro de la filosofía del lenguaje durante el siglo XX y lo que va del presente siglo. Dicha discusión apunta al hecho de -cómo se entiende el significado-, ya sea ligado al significado léxico dado, o en la interacción entre la lengua y el uso, tal situación aún no ha sido finiquitada de una forma aceptada por todos los investigadores de la filosofía del lenguaje (Cornejo, 2004).

Las clasificaciones de actos de habla tanto de Austin como de Searle pueden ser claves en la búsqueda de un anclaje entre actos de habla y semántica, lo cual puede servir en parte para entender la problemática del significado, en particular a partir de los criterios utilizados por cada uno de estos filósofos para elaborar sus clasificaciones de actos ilocucionarios.

Dado el interés que pueden aportar los planteamientos de estos investigadores proponemos dar cuenta del tratamiento que cada uno otorga, tanto al aspecto semántico como al aspecto acto de habla en la búsqueda de conciliación entre estos dos niveles o aspectos.

Para dar cuenta de este problema este artículo abordará los elementos que consideran Austin y Searle para constituir sus categorías de verbos ilocucionarios, para ir en búsqueda de la idea de significado que sustentan en sus respectivas taxonomías. Este propósito implica la revisión de conceptos como ilocución y fuerza ilocucionaria que utilizan estos autores. Luego, se presentan las propuestas de verbos y actos ilocucionarios y los respectivos criterios utilizados para su elaboración por cada investigador. Finalmente, a partir de la revisión de las clasificaciones se discute la idea de significado que sustenta cada autor. 


\section{Marco Teórico}

Mencionaremos el conflicto del significado entre semántica y pragmática; luego revisaremos las definiciones y clasificación de actos ilocucionarios, primero de Austin y luego de Searle.

Como planteáramos en la introducción, la discusión sobre los límites del significado es un tema aún no zanjado, ya que coexisten al menos dos maneras de entender el significado. Para algunos autores la semántica es el estudio del significado lingüístico ligado al significado léxico dado; para otros investigadores como Wittgenstein, Austin, Searle y Grice, entre otros, es necesario incorporar el uso de la lengua en el significado (Cornejo, 2004).

De la segunda perspectiva, entre la estructura y el uso de la lengua surge la pragmática. Dicha disciplina apunta al estudio del significado de las ilocuciones lingüísticas en la interacción de los hablantes de una lengua. Por ello, el aspecto pragmático del significado lingüístico es entendido como experiencia subjetiva, por lo tanto, el estudio del uso de la lengua tiende a ir más allá de la descripción estructural del significado en sí mismo y centra la atención en la comprensión subjetiva de las ilocuciones (Cornejo, 2004).

Cornejo (2004) sostiene que gran parte de la investigación que se puede clasificar hoy como pragmática asume, implícita o explícitamente una definición del significado lingüístico ajena a la experiencia del significado. En dicha perspectiva los aspectos pragmáticos se reducen a las inferencias que son subsidiarias y complementarias al significado en sí mismo del léxico mental que continúa siendo considerado como el significado lingüístico verdadero. Para Cornejo (2004) la pragmática clásica no ha abandonado la opinión estructuralista del significado, esto debido a la falta de conciliación entre la semántica y la pragmática.

A partir de lo sostenido por Cornejo (2004) con respecto a la pragmática clásica podemos considerar que la idea de clasificar actos de habla de Austin (1996) y Searle (1991b) sigue vigente. La pregunta que posiblemente debieron tratar de responder estos autores debe haber similar a: ¿cómo identificamos los valores de significado subjetivo de expresiones lingüísticas que se ven modificadas por el uso?

Un punto a considerar antes de la revisión conceptual es que la construcción de la "pragmática" como nueva disciplina o área de la lingüística toma su conceptualización a partir de la adaptación y traslado de conceptos que originalmente fueron creados para la filosofía del lenguaje y que en muchos casos pasan a la lingüística sin una clarificación ni adecuación (Vallejos, 1987). 


\subsection{Conceptos de Austin}

Un primer elemento a considerar quizá como un principio de Austin (1996) es que "decir es hacer", es decir, la emisión de una expresión lingüística produce una acción en el mundo, por cuanto los enunciados performativos o realizativos ejercen una acción en el mundo.

Austin (1996) comienza sus conferencias aclarando que existen enunciados que no son descriptivos, y por tanto no pueden calificarse como verdaderos o falsos, es decir, existen enunciados que no describen la realidad, ni pueden evaluarse en términos de verdad o falsedad. Estos enunciados al ser pronunciados hacen o ejecutan la acción.

Los verbos que participan de este tipo de enunciados tienen por característica básica no describir, ni registrar ningún hecho, no son verdaderos, ni falsos y al ser expresados en una oración la acción se ejecuta. Para Austin (1996: 47): "emitir la expresión es realizar una acción". Agrega el autor: "al emitir nuestros realizativos estamos sin duda, y en un sentido correcto, "realizando acciones"". (Austin, 1996: 52).

Para Austin (1996: 66) una expresión realizativa es:

"aquella expresión lingüística que no consiste, o no consiste meramente, en decir algo, sino en hacer algo, y que no es un informe, verdadero o falso, acerca de algo".

Agrega Austin (1996: 49):

"expresar las palabras es, sin duda, por lo común, un episodio principal, sino el episodio principal, en la realización del acto, cuya realización es también la finalidad que persigue la expresión".

Considera además Austin: "siempre es necesario que las circunstancias en que las palabras se expresan sean apropiadas, de alguna manera" (Austin, 1996: 49)

Para que una acción se ejecute exitosamente además del hecho de pronunciarlas es requisito que otros aspectos sean considerados (Austin, 1996). Tales aspectos a estimar son analizados por Austin (1996) y a partir de ellos el autor elabora su teoría de los infortunios. Esta teoría corresponde a una serie de condiciones que debe cumplir un enunciado realizativo para ser considerado satisfecho, de no cumplir tales condiciones el enunciado no logra ser un realizativo. 
Al finalizar la conferencia IV Austin (1996) se propone distinguir los enunciados constatativos de los realizativos. En esta búsqueda de un criterio de identificación entre ambos tipos de enunciados surgen los realizativos primarios y los realizativos explícitos. Los primarios corresponden a la base realizativa que está implícita; los explícitos corresponden a los enunciados realizativos directos. Como en el ejemplo que ofrece Austin (1996): 1) expresión primaria: "estaré allí", 2) realizativo explícito: "le prometo que estaré allí".

Según Austin (1996) el realizativo explícito es el resultado de la evolución del realizativo primario. El lenguaje como tal, en sus estadios primitivos, no es preciso, y tampoco es explícito en el sentido que estamos dando a esta última palabra.

La expresión de un enunciado se estructura en tres tipos de actos simultáneos, el acto locucionario, el acto ilocucionario y el acto perlocucionario. El acto locucionario corresponde a la realización de una emisión, es realizar una ilocución. Para determinar el acto ilocucionario hay que determinar el uso de la locución. La locución presenta fuerza ilocucionaria, la de realizar un acto o la de llevar a cabo el acto de decir algo.

Austin (1996) sostiene que los filósofos se han desentendido del estudio y tratan todos los problemas como problemas de uso locucionario.

En la conferencia IX Austin concluye que el divorcio entre las acciones físicas y los actos de decir algo no es del todo completo: hay vinculación, por cuanto "el acto ilocucionario es un acto convencional" (Austin, 1996: 197).

\subsection{La clasificación de fuerzas ilocucionarias de Austin}

Austin (1996: 197) propone "una lista de fuerzas ilocucionarias de una expresión". Considera que los test para identificar los realizativos explícitos son adecuados para identificar los verbos que "explicitan la fuerza ilocucionaria de una expresión" o "ponen de manifiesto cuál es el acto ilocucionario que estamos realizando al emitirla" (Austin, 1996: 197), es decir, a Austin le interesa identificar qué actos ilocucionarios se realizan al emitir determinados verbos.

En relación al test planteado como criterio de identificación de fuerzas ilocucionarias o acto ilocucionario Austin (1996: 197-198) sostiene que:

“Usaremos pues, con cautela, el test simple de la primera persona del singular del presente del indicativo en la voz activa, y recorreremos el diccionario animados por un es- 
píritu liberal. Obtendremos una lista de verbos en el orden de 10 a la tercera potencia."

El acto ilocucionario se realiza en la interacción entre hablantes al pronunciar determinadas expresiones lingüísticas que explicitan dichas fuerzas ilocucionarias.

Austin (1996) plantea así su propuesta:

"Clasificaré estas expresiones en función de sus fuerzas ilocucionarias, y les asignaré estos nombres:

1) verbos de judicación, o judicativos;

2) verbos de ejercicio, o ejercitativos;

3) verbos de compromiso, o compromisorios;

4) verbos de comportamiento, o comportativos; $y$

5) verbos de exposición, o expositivos.

Austin (1996:199) sostiene que: "debemos tener claro desde el comienzo que quedan amplias posibilidades de que se presenten casos marginales o difíciles, así como superposiciones". Y, agrega: "En modo alguno estoy proponiendo nada definitivo".

El foco de Austin (1996) es la clasificación de expresiones en función de sus fuerzas ilocucionarias.

Tabla No1 A partir de Austin, 1996

\begin{tabular}{|c|c|c|}
\hline Nombre & Acto típico & Características \\
\hline Judicativos & $\begin{array}{l}\text { Emitir un veredicto o } \\
\text { juicio sobre algo }\end{array}$ & $\begin{array}{l}\text { El rol social del hablante debe facultarlo para } \\
\text { dictaminar o arbitrar a través de un juicio. }\end{array}$ \\
\hline Ejercitativos & $\begin{array}{l}\text { Ejercicios de poder, } \\
\text { derechos o influencia }\end{array}$ & $\begin{array}{l}\text { Se decide sobre la conducta pasada o futura de } \\
\text { otros, en orden a realizar ciertos actos. }\end{array}$ \\
\hline Compromisorios & $\begin{array}{l}\text { Prometer o } \\
\text { comprometer a hacer } \\
\text { algo. }\end{array}$ & $\begin{array}{l}\text { El hablante se compromete a cierta línea de } \\
\text { acción sobre acciones futuras o consecuencias } \\
\text { supuestas. }\end{array}$ \\
\hline Comportativos & $\begin{array}{l}\text { Actitudes y } \\
\text { comportamiento } \\
\text { social }\end{array}$ & $\begin{array}{l}\text { Son una reacción frente a la conducta o } \\
\text { condición de otros, o bien pueden ser } \\
\text { actitudes frente a la conducta pasada o } \\
\text { inminente de otros. }\end{array}$ \\
\hline Expositivos & $\begin{array}{l}\text { Modo como } \\
\text { expresiones, encajan } \\
\text { en un argumento o } \\
\text { conversación }\end{array}$ & $\begin{array}{l}\text { Son recursos que utiliza un expositor para } \\
\text { expresar opiniones, conducir debates y } \\
\text { clarificar usos y referencia. }\end{array}$ \\
\hline
\end{tabular}

\subsection{Conceptos de Searle}

Para Searle (1991a: 432) el acto ilocucionario: "es la unidad mínima de la comunicación lingüística" (...) "la comunicación lingüística incluye 
esencialmente actos", es decir, la base del significado de la comunicación lingüística estaría en el acto ilocuionario, a través de los cuales nos comunicamos.

Para Searle (1991a) "una proposición no es un acto aunque el acto de expresar una proposición sea una parte de la realización de ciertos actos ilocucionarios". El significado no está en la proposición, sino en el acto que la expresa, por cuanto este autor focaliza la base del significado en el acto ilocucionario.

La realización de actos obedece a un tipo de conducta, para Searle (1991a: 432): "realizar un acto ilocucionario es tomar parte de una forma de conducta gobernada por reglas", es decir, conocer los significados es conocer las reglas para su uso, y no el significado anclado sólo en el nivel léxico de las palabras empleadas en las proposiciones.

Searle (1991a) reconoce que otros filósofos han negado el punto de vista de que el significado es un asunto de reglas y han aseverado que no existen en absoluto reglas semánticas del género propuesto.

Para Searle (1991a) existen dos clases de reglas semánticas, las reglas que regulan las formas de conducta existentes y las reglas que definen nuevas formas de conducta.

Una distinción importante en Searle (1991b) es la que opera entre un acto ilocucionario y el contenido proposicional. Para este autor desde un enfoque semántico una oración posee un indicador proposicional y un indicador de fuerza ilocucionaria. El primero corresponde al indicador de proposición, es decir, la proposición misma; y el segundo, contiene el indicador de función, es decir, al acto de habla en cuestión.

El significado no es para Searle (1991a) un asunto de intención sino que "es un asunto de convención" (Searle, 1991a: 440), es decir, para que la expresión de una emisión produzca un acto, este acto debe estar sujeto a reglas convencionalizadas y no pretender que con la sola intención del hablante se manifieste el significado de cualquier acto que no esté sujeto a reglas.

No obstante lo anterior, según Searle (1991a: 440) “En nuestro análisis de los actos ilocucionarios debemos capturar tanto los aspectos convencionales como los intencionales y especialmente las relaciones entre ellos", es decir, es necesario establecer un puente entre el significado intencional que manifiesta un sujeto al pretender comunicarse y el uso que este sujeto realiza, a través del manejo de las reglas de uso de una lengua y que se manifiesta en las expresiones convencionalizadas por el uso. 
Para Searle (1991a), al realizar un acto ilocucionario el hablante intenta producir un cierto efecto logrando que el oyente reconozca su intención de producir ese efecto, y además, si está usando las palabras literalmente, intenta que este reconocimiento se logre en virtud del hecho que las reglas para el uso de las expresiones que emite asocien las expresiones con la producción de ese efecto.

Para dar cuenta de las reglas que participan del uso de un acto ilocucionario, Searle (1991b) analiza el verbo prometer a la luz de 12 condiciones, con el fin de obtener de dicho ejercicio la base para formular reglas que sean comunes a todos los actos ilocucionarios. A partir de esto extrae las condiciones que corresponden al indicador de función y que son aplicables a todos los tipos de actos ilocucionarios.

Searle (1991b: 450) tiene muy claro que: "los verbos ilocucionarios son parte de un lenguaje particular: francés, alemán, castellano, o cualquier otro", y que : "las ilocuciones son parte del lenguaje como opuesto a los lenguaje particulares", por ello considera que Austin (1996) se equivoca al equiparar verbos ilocucionarios con actos ilocucionarios.

En el mismo sentido, Searle (1991b) considera que los verbos pudiendo ser una buena guía de identificación de actos ilocucioanrios, no sirven para establecer diferencias en los actos ilocucionarios. Ya que, en casos como los actos de petición y orden, que comparten el objeto ilocucionario, el cual consiste en "el intento de lograr que los oyentes hagan algo", difieren en cuanto a su fuerza ilocucionaria. La petición apunta a una solicitud de acción favorable al hablante y en la que la posición de ambos interactuantes puede ser más bien simétrica, por cuanto no hay obligatoriedad de la acción a solicitar al oyente; en cambio, la orden apunta a una relación más bien asimétrica en que se manifiesta la obligatoriedad de la acción hacia el oyente. Por consiguiente para Searle (1991b) el "objeto ilocucionario" no es lo mismo que la "fuerza ilocucionaria", si bien el "objeto ilocucionario" es parte de la "fuerza ilocucionaria".

\subsection{Categorías de actos ilocucionarios de Searle}

La primera condición o criterio aplicable a todo acto ilocucionario considerada por Searle (1991b) será la que distingue el objeto ilocucionario de la fuerza ilocucionaria. En el análisis la fuerza ilocucionaria de la emisión se codificará como (F) y su contenido proposicional como (p). El propósito de Searle es clarificar los diferentes tipos de fuerza ilocucionaria, que pueden aislarse del contenido proposicional.

Es importante considerar que no toda expresión puede tener un contenido proposicional, pero si toda expresión posee una fuerza ilocu- 
cionaria. Como el caso de la expresión: ¡Hurra!, que constituye un tipo de acto ilocucionario que carece de contenido proposicional.

Un segundo criterio lo constituye la dirección de ajuste entre palabras y el mundo, la cual considera dos posibilidades, la primera es que las palabras encajen con el mundo y la segunda es que el mundo encaje con las palabras. Las aserciones corresponderían al primer tipo y las promesas y ruegos corresponderían al segundo. La dirección de ajuste es una consecuencia del objeto ilocucionario.

Un tercer criterio corresponde a las diferencias en los estados psicológicos expresados. Ya que, en la realización de cualquier acto ilocucionario con un contenido proposicional, el hablante expresa alguna actitud o estado psicológico hacia ese contenido proposicional. El estado psicológico expresado en la realización de acto ilocucionario es la condición de sinceridad del acto.

Estas tres dimensiones o criterios, que en la práctica constituyen cuatro elementos: objeto ilocucionario, contenido proposicional, dirección de ajuste y condición de sinceridad (Ver cuadro № 1) son la base de la clasificación o taxonomía de actos ilocucionarios propuesta por Searle (1991b).

Cuadro № 1 Basado en Searle, 1991b

\section{Dimensiones o criterios de Searle, 1991}

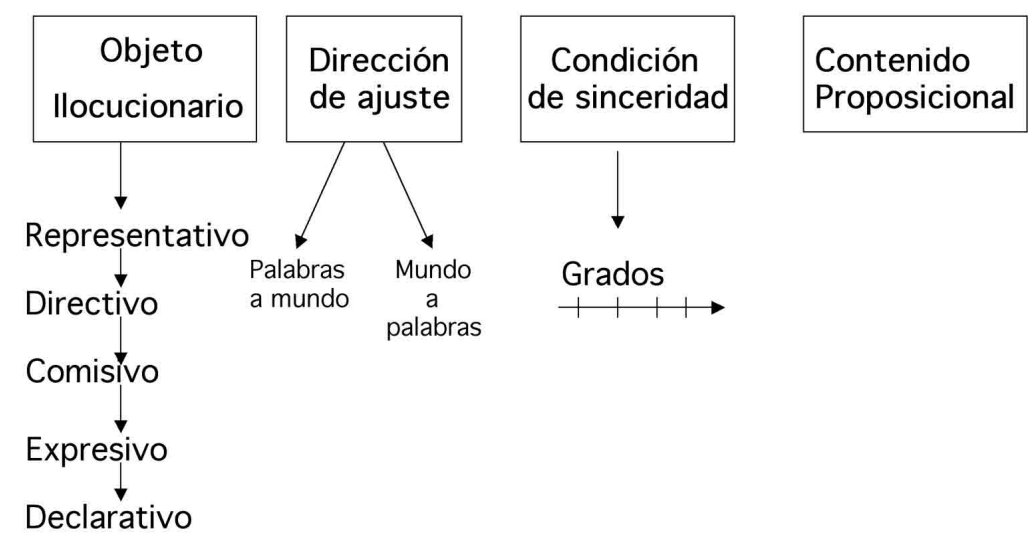

1. asertivos o representativos

2. directivos

3. comisivos

4. expresivos

5. declarativos 
Austin y Searle: la relación entre los verbos y actos ilocucionarios /

Marco Alarcón Silva

Tabla No 2 A partir de Searle, 1991b

\begin{tabular}{|l|l|l|l|}
\hline Nombre & Propósito & Mundo-Lenguaje & Sinceridad \\
\hline Representativos & $\begin{array}{l}\text { Comprometer al hablante } \\
\text { con la verdad de la } \\
\text { proporción expresada }\end{array}$ & $\begin{array}{l}\text { La palabra se } \\
\text { ajusta al mundo y } \\
\text { es verdad o no. }\end{array}$ & $\begin{array}{l}\text { Tales actos se creen. } \\
\text { Son evaluables } \\
\text { según valoración } \\
\text { Verdadero o Falso. }\end{array}$ \\
\hline Directivos & $\begin{array}{l}\text { Intentar que el oyente } \\
\text { haga algo }\end{array}$ & $\begin{array}{l}\text { El mundo es el } \\
\text { que se ajusta al } \\
\text { lenguaje }\end{array}$ & $\begin{array}{l}\text { Conducen estado de } \\
\text { deseo. }\end{array}$ \\
\hline Comisivos & $\begin{array}{l}\text { Comprometer al hablante } \\
\text { en una acción futura }\end{array}$ & $\begin{array}{l}\text { El mundo se ajusta } \\
\text { al lenguaje }\end{array}$ & $\begin{array}{l}\text { El hablante debe } \\
\text { tener la intención } \\
\text { o bien actuar como } \\
\text { dice. }\end{array}$ \\
\hline Expresivos & $\begin{array}{l}\text { Expresar el estado } \\
\text { psicológico del hablante, } \\
\text { especificado en la } \\
\text { condición de sinceridad } \\
\text { sobre el estado de } \\
\text { cosas especificado en el } \\
\text { contenido proposicional. }\end{array}$ & $\begin{array}{l}\text { No hay dirección } \\
\text { de ajuste. }\end{array}$ & $\begin{array}{l}\text { Corresponderá al } \\
\text { estado psicológico } \\
\text { del hablante. }\end{array}$ \\
\hline Declarativos & $\begin{array}{l}\text { Modificar una situación, } \\
\text { creando una nueva }\end{array}$ & Relación recíproca \\
quien habla.
\end{tabular}

\section{Metodología}

A partir de la revisión conceptual del marco teórico discutiremos las propuestas de Austin y Searle en relación a la idea de significado subyacente a la vinculación entre actos de habla y semántica desde las clasificaciones de actos ilocucionarios.

\section{Discusión}

En relación a la propuesta de Austin (1996) no podemos considerar que el criterio del test y la búsqueda de diccionario sea un criterio de clasificación propiamente tal, sí podemos considerarlo un método de búsqueda, pero no nos entrega información sobre cómo agrupar o categorizar los actos ilocucionarios. Por ello debemos intentar inferir la idea de significado de Austin a partir de la clasificación y relación general que establece con respecto a los actos de habla y los verbos.

Habiendo considerado lo planteado en el párrafo anterior sostenemos que Austin (1996) pretende explicar "cuando decir X significa hacer Y". Su foco está más en las "características" de la clasificación de fuerzas ilocucionarias, que en "fijar" un verbo a una única clasificación, es decir, cuando 
un verbo se corresponde con determinado acto ilocucionario. Por ello, la locución de un mismo verbo, puede participar del significado de más de una fuerza ilocucionaria a la vez, dependiendo de ciertas condiciones o características de la emisión de dicha locución.

Una misma expresión puede servir para hacer distintos actos, ya que puede modificarse la fuerza ilocucionaria. Además, si bien se ha criticado a Austin por equiparar fuerza ilocucionaria con expresión (Searle, 1991b, 1994), sus dudas y comentarios previos a la propuesta de "listado de fuerzas ilocucionarias" dejan entrever que él considera la posibilidad de la superposición, pero no sostiene que la relación entre acto ilocucionario y verbo sea uno a uno, como plantea Searle (1991b) en sus críticas a Austin.

Probablemente la confusión de Austin (1996) entre categorías que se superponen provenga del hecho de que una misma expresión puede ser utilizada en distintas interacciones y si además consideramos que el significado de una expresión puede "enriquecerse" en diversas condiciones, el valor de una misma expresión puede variar, adquiriendo distintos significados.

En otras palabras Austin (1996) busca un anclaje en "listas de verbos" de las fuerzas ilocucionarias, resultando entonces que una misma expresión -verbo de la lista- puede corresponder a más de una categoría de fuerza ilocucionaria.

En este mismo sentido podemos cuestionar entonces ¿qué aspectos son los que modifican a una "expresión $X$ " a significar en ciertas interacciones "hacer $Y$ " o "hacer $Z$ ".?. Entre " $X$ ", que en la "interacción 1 vale hacer Y"; $y$ " $X$ " que en la "interacción 2 vale hacer $Z$ ". ¿Qué es lo que hace que " $X$ " en la "interacción 1" tenga un valor distinto a " $X$ " en la "interacción 2"? Si pensamos que es sólo un valor asociado a la expresión " $X$ ", confundiremos los verbos de una lengua como si fuesen universales, $y$ efectivamente estaremos equiparando un verbo de una lengua natural con un acto ilocucionario que es universal; o bien, si consideramos que la modificación del significado corresponde a un valor asociado a la interpretación que se considera convencionalizada (Austin, 1996) y que además entenderemos como subjetiva (Cornejo, 2004). Se produce entonces que el significado de la misma expresión difiere entre una "interacción 1" y una "interacción 2", portando valores distintos.

El hecho que hayan superposiciones entre expresiones que aparecen entre una y otra categoría demuestra que Austin al forzar la expresión y tratar de obtener -expresiones- que correspondan sólo a una clase o categoría de fuerza ilocucionaria, genera la ambigüedad entre las catego- 
rías. Además, si consideramos que lo superpuesto no son las categorías de fuerzas ilocucionarias, sino los significados de ciertos verbos, podemos explicar el porqué se modifica su valor como fuerza ilocucionaria.

Es posible afirmar, entonces, que el valor de una fuerza ilocucionaria no está fijo a una determinada expresión. Quizá el error de Austin esté justamente en este punto, al focalizar el valor de las fuerzas ilocucionarias en las expresiones como pertenecientes a categorías, es decir, al focalizarse en la categoría genera la confusión y la ambigüedad y por ende la inestabilidad de su taxonomía.

Dicho de otra forma, el error de Austin puede ser el conectar las categorías y relacionarlas, en vez de relacionar las fuerzas ilocucionarias presentes en una misma expresión y que en ese sentido pueden formar parte de distintas categorías de fuerzas ilocucionarias, pero no de verbos ilocucionarios.

Una posible corrección de la clasificación de Austin debiera tener en cuenta que si el significado de la fuerza ilocucionaria de un verbo se modifica por interacción -el criterio debiera ser ubicar las fuerzas ilocucionarias prototípicas, describir situaciones e interacciones y luego buscar la posibilidad de categorizar las fuerzas ilocucionarias-. Las condiciones lógicas posiblemente emanadas desde este punto pueden ser la base de una categorización y no los verbos, ya que así un mismo verbo si cumple con " $X$ " condiciones de fuerza ilocucionaria en ciertas situaciones puede pertenecer a una u otra categoría de fuerza ilocucionaria y no estar determinado a una clase.

Si consideramos que el significado de la fuerza ilocucionaria se enriquece en la interacción, lo que se modifica son las interacciones, lo que a su vez modifica el significado de una misma expresión. Entonces, el significado no está al parecer completamente dado sólo en la expresión, sino que la interacción modifica el significado.

También, podemos sostener que existe parte del significado de la fuerza ilocucionaria que sí está en la expresión y que luego se enriquece en la interacción, o ¿es sólo el significado de la interacción el que vale?. Además, es posible que no ocurra lo mismo con todos los verbos, lo que complejiza aún más las posibles reglas que soporten las relaciones entre los actos ilocucionarios y la semántica de los verbos.

Por otro lado, existe una condición implícita de Austin, la cual consiste en considerar los actos de habla en situaciones ritualizadas instaladas en la sociedad, lo cual puede verse muy claramente en las caracterizaciones de los tipos de fuerzas ilocucionarias. De manera que no están presentes en su clasificación las situaciones cotidianas. 
Además, el foco de su clasificación está en los realizativos explícitos, ya que considera a los realizativos primarios como actos de habla indirectos, a través de los cuales una misma fuerza ilocucionaria puede ser generada de manera indirecta. Lo cual dificulta aún más la pretensión de la categorización.

En cuanto a la taxonomía de actos ilocucionarios propuesta por Searle (1991b), diversos autores consideran que es un paso adelante en relación a la clasificación de Austin (Vallejos, 1987; Bertuccelli, 1996; Escandell, 2003; Charaudeau y Maingueneau, 2005).

Searle (1991b) sostiene que no intentó clasificar verbos ilocucionarios, dado que sus criterios o dimensiones abordan cuatro puntos claves: el objeto ilocucionario, la dirección de ajuste, la condición de sinceridad y el contenido proposicional. Tales criterios le permiten plantear que no se debe confundir nombres de verbos que pueden ser ilocucionarios con nombres de géneros ilocucionarios.

No obstante, el avance que puede significar la propuesta de Searle en relación a Austin, existen investigadores como Sadock (1994) quien considera que algunos de los aspectos de la clasificación de Searle están fuera de foco, como por ejemplo la dimensión "dirección de ajuste" entre las palabras y el mundo, la cual no tendría ningún sentido de realidad, independiente de la estructura de los actos de habla. La crítica de Sadock (1994) apunta al hecho de no encontrar fundamento en Searle (1991b) para sostener que ciertos actos de habla son primitivos de los que se supone son indirectos.

Por otro lado, el propio Sadock (1994) plantea que no puede ser mera coincidencia que la taxonomía de Austin (1996) presente cinco clases de ilocucionarios al igual que la de Searle (1991b). (Ver la siguiente tabla).

Tabla № 3 A partir de Sadock, 1994

\begin{tabular}{|c|c|c|c|}
\hline (Austin, 1996) & \multirow[t]{6}{*}{ Criterio Ambiguo } & (Searle, 1991, 1994) & \multirow{6}{*}{$\begin{array}{l}\text { 1. Coincide con Autin de } \\
\text { 1-4; } 5 \text { refinamiento de } 5 \text { en } \\
\text { Austin. } \\
\text { 2. Fuera de Foco. Desajuste } \\
\text { entre palabras y el mundo. } \\
\text { No hay sentido de realidad. } \\
\text { 3. Valores arbitrarios, ¿de } \\
\text { dónde saca los primitivos?, } \\
\text { porque no considera actos } \\
\text { como maldecir, quejarse y } \\
\text { suponer. }\end{array}$} \\
\hline 1. Veridictivos & & 1.Asertivos & \\
\hline 2. Ejercitativos & & 2.Directivos & \\
\hline 3. Comisivos & & 3.Comisivos & \\
\hline 4. Conductuales & & 4.Expresivos & \\
\hline 5. Expositivos & & 5. Declarativos & \\
\hline
\end{tabular}

Para Sadock (1994), la taxonomía de Austin presenta un criterio ambiguo. Searle (1991b) sostiene que su clase asertiva contendrá la mayoría 
de los expositivos de Austin y muchos de sus veridictivos. Y, muchos ejercitativos de Austin son también de esta clase. Los actos de agradecimiento, de disculpas y de condolencias ocurren en la lista de Austin de los conductuales y son incluidos entre los expresivos de Searle. La categoría expositiva es absorbida casi en su totalidad dentro de los asertivos de Searle. No obstante las posibles relaciones que se puedan establecer, Sadock (1994) señala que las cinco categorías difieren completamente entre uno y otro autor.

Independiente de las críticas que pueda recibir la propuesta de Searle (1991b) es indudable que la formalización a partir de las cuatro dimensiones de análisis de una expresión, permite visualizar de forma muy adecuada la idea de varios continuum que constituyen los actos ilocucionarios, lo cual es un avance en relación a la metáfora de la fuerza ilocucionaria de Austin (1996) que sugería que las diferentes fuerzas ilocucionarias ocupaban posiciones diferentes en un único continuum de fuerza.

Searle (1991b: 450) sostiene que: "lo que efectivamente sucede es que existen varios continuos distintos entrecruzados", es decir, no es posible igualar verbo ilocucionario y acto ilocucionario, por cuanto "las ilocuciones son parte del lenguaje como opuesto a los lenguaje particulares".

El significado de los actos ilocucionarios corresponde claramente a una consideración universal, y los verbos son realizaciones particulares dentro de una determinada lengua. Lo interesante es entonces detectar cuales son las reglas que subyacen al uso de determinados verbos que se conectan con determinados actos y producen una modificación de significado.

Si bien existe indudablemente un significado léxico asociado a verbos que pueden estar en el diccionario, este significado no está completo, y no es posible obtenerlo en relación uno a uno con respecto a los actos de habla. La dificultad radica entonces en detectar teóricamente como se vincula el continuum de actos ilocucionarios con verbos que en diversas interacciones producen determinados actos ilocucionarios.

\section{Conclusión}

Esta investigación consistió en intentar dar cuenta de la idea de significado que subyace en la relación entre actos de habla y verbos, o también podemos decir, entre la semántica y la pragmática. Para ello revisamos algunos conceptos que consideramos importantes en Austin y Searle y que vinculamos a las clasificaciones de actos ilocucionarios que propone cada uno, en particular en relación a los criterios utilizados para abordar el problema. 
Podemos identificar que existen reglas que sustentan las convenciones entre los significados que poseen los verbos y que a través de la interacción pragmática constituyen su significado. Por cuanto el significado de los verbos no depende solamente de su constitución léxica dada, sino que se potencia dicha constitución léxica en la interacción o uso de una expresión en una emisión real, en que se constituye como acto ilocucionario.

La intención que si bien está presente en el nivel pragmático es una dimensión que aporta significado, pero que sin una convención no se logra una comunicación o intersubjetividad.

Los actos ilocucionarios constituyen varios continuum de fuerza ilocucionaria y no un único patrón de continuum.

Los aportes de Austin son fundamentales para constituir la relación entre la lengua y el uso. La confusión que puede haber generado la relación entre las categorías de actos y los verbos debe ser superada y entender la globalidad de la propuesta de Austin en la cual su aporte al desarrollo de la pragmática es vital.

La formalización de Searle en las cuatro dimensiones es muy interesante, el análisis del contenido proposicional y la conexión con la fuerza ilocucionaria permite estructurar el análisis, aún cuando puede llevar la discusión a la sintaxis y alejarla de la semántica. Incluso es posible vincular esta propuesta con la idea de estructura de superficie y estructura profunda de Chomsky.

Un aporte importante de este trabajo puede ser en particular la discusión sobre la propuesta de Austin vista desde una perspectiva más analítica. La principal debilidad puede ser la amplitud de la problemática que cuesta situar.

Puede ser importante continuar la revisión con otras categorizaciones de los actos de habla y plantear revitalizadamente la discusión sobre el significado enriquecido por la interacción en el uso de la lengua.

\section{Referencias Bibliográficas}

Austin, J. (1996)

Bertuccelli, M. (1996)

Cornejo, C. (2004)
¿Cómo hacer cosas con palabras? Madrid: Paidós.

Qué es la pragmática. Barcelona: Paidós.

"Who says what the words say? Theory \&

Psychology". Sage Publications. Vol. 14 (1) :5-28. 
Austin y Searle: la relación entre los verbos y actos ilocucionarios /

Marco Alarcón Silva

Charaudeau, P. \& Maingueneau, D. (2005)

Diccionario de análisis el discurso. Buenos Aires: Amorrortu.

Escandell, M (2003)

Introducción a la pragmática. Madrid: Ariel.

Sadock, J. (1994)

"Toward a grammatically realistic typology of speech acts".393-406. En L. Savas (edit.) Foundations of speech act theory. London: Routledge.

Searle, J. (1994)

Actos de habla. Madrid: Cátedra.

Searle, J. (1991a)

"¿Qué es un acto de habla?". En L. Valdés (edit.) La búsqueda del significado. pp. 431-476. Madrid: Ed.Tecnos.

Searle, J. (1991b)

"Una taxonomía de los actos ilocucionarios". En L. Valdés (edit.) La búsqueda del significado. pp. 431476. Madrid: Ed.Tecnos.

Vallejos, G. (1987)

"Algunas bases filosóficas de la pragmática lingüística". Lenguas Modernas 14, 5-56. 